\title{
Standing kink oscillations of thin twisted magnetic tubes with continuous equilibrium magnetic field
}

\author{
M. S. Ruderman ${ }^{1,2}$ and J. Terradas ${ }^{3}$ \\ ${ }^{1}$ Solar Physics and Space Plasma Research Centre ( $\left.\mathrm{SP}^{2} \mathrm{RC}\right)$, University of Sheffield, Hicks Building, Hounsfield Road, \\ Sheffield S3 7RH, UK \\ e-mail: m.s.ruderman@sheffield.ac.uk \\ 2 Space Research Institute (IKI) Russian Academy of Sciences, 117342 Moscow, Russia \\ 3 Departament de Física, Universitat de les Illes Balears, 07122 Palma de Mallorca, Spain
}

Received 23 March 2015 / Accepted 4 June 2015

\begin{abstract}
In this article we study standing kink waves in twisted magnetic tubes. We use the cold plasma and thin tube approximation. We assume that the plasma density is constant inside and outside the tube. We also assume that the magnetic twist is weak and take the ratio of the azimuthal and axial component of the magnetic field to be of the order of ratio of the tube radius and tube length. The azimuthal component of the magnetic field is proportional to the distance from the tube axis inside the tube, and inversely proportional to this distance outside the tube. Using the method of asymptotic expansions we derived the governing integral equation that determines the eigenfrequencies and eigenmodes of the tube kink oscillations. In the approximation of a very weak twist, we calculated analytically the corrections to the frequencies of the fundamental mode and first overtone of a straight magnetic tube related to the presence of twist. The analytical results are compared with the numerical results obtained using the full set of linear ideal magnetohydrodynamic equations. We also calculated the ratio of frequencies of the fist overtone and fundamental mode. We found that the magnetic twist enhances this ratio for moderate values of the density ratio, and reduces this ratio for large values of the density ratio. In general, the deviation of the frequency ratio from 2 caused by the magnetic twist is comparable to that found in simultaneous observations of the fundamental mode and first overtone of the coronal loop kink oscillations. Finally, we studied the eigenmode polarization. We found that, in a particular case of linear polarization, the polarization direction rotates along the tube.
\end{abstract}

Key words. hydrodynamics - magnetohydrodynamics (MHD) - plasmas - waves

\section{Introduction}

Since transverse oscillations of coronal magnetic loops were first detected by TRACE and interpreted as fast standing kink waves (Aschwanden et al. 1999; Nakariakov et al. 1999), they continue to enjoy ample attention of solar physicists. In the first theoretical interpretation of this phenomenon a very simple model of a coronal magnetic loop, which is a straight magnetic tube, was used (e.g. Ryutov \& Ryutova 1976; Edwin \& Roberts 1983). Later, more sophisticated models have been developed. For a review of the theory of coronal loop oscillations, see for example Ruderman \& Erdélyi (2009).

One important problem in the theory of coronal loop kink oscillations is the effect of magnetic twist. Twisted magnetic tubes have been studied for many years in the context of the tube stability (e.g. Dungey \& Loughead 1954; Roberts 1956; Shafranov 1957; Kruskal \& Tuck 1958; Parker 1974; Browning \& Priest 1983). Twisted magnetic tubes have also been studied in relation to the magnetohydrodynamic (MHD) wave resonant absorption (e.g. Sakurai et al. 1991; Goossens \& Ruderman 1995; Goossens et al. 1995, 2011; Ballai \& Erdélyi 2002). Wave propagation in twisted magnetic tubes has been investigated by Bogdan (1984), Bennett et al. (1999), Erdélyi \& Carter (2006), Carter \& Erdélyi (2007, 2008), Erdélyi \& Fedun (2007), and Terradas \& Goossens (2012).

To apply this to the coronal loop kink oscillations it is important to study standing waves in twisted magnetic tubes. An equilibrium that consists of a straight magnetic tube with purely axial magnetic field is mirror symmetric with respect to a plane orthogonal to the magnetic field lines. As a result, the properties of propagating waves are independent of the propagation directions. In that case a standing wave can be constructed as a superposition of two propagating waves with the same frequencies and wave numbers that propagate in the opposite directions. The situation changes when there is the magnetic twist because it destroys the mirror symmetry of the equilibrium. For particular equilibria with the twisted magnetic field it is still possible to obtain a standing wave as a superposition of two propagating waves with the same frequencies, but now with different wavenumbers (see e.g. Ruderman 2007, Paper I). However, in general, a solution describing a standing wave in a twisted tube cannot only be obtained as a superposition of two, but even of a few propagating waves. Hence, the investigation of standing waves in a twisted magnetic tube is very much different from the investigation of propagating waves.

In Paper I, we considered standing kink waves in a thin twisted magnetic tube with a purely axial magnetic field outside the tube and azimuthal component of the magnetic field proportional to the radial distance from the tube axis inside the tube. Karami \& Bahari (2010) numerically studied the effect of magnetic twist on the resonant damping and the period ratio of the fundamental harmonic and first overtone in a magnetic tube. They considered an equilibrium with the azimuthal component of the magnetic field proportional to the distance from the tube axis. Hence, this component magnitude tends to infinity 
when the distance from the tube axis increases. These equilibrium configurations can hardly be considered a realistic model of a coronal loop. Karami \& Bahari (2012) investigated the effect of magnetic twist on the period ratio of the fundamental harmonic and first overtone in a magnetic tube using the thin tube and cold plasma approximation. They considered an equilibrium in which the azimuthal component of the magnetic field is proportional to the distance from the tube axis in the tube core and in the surrounding annulus, the proportionality coefficients being different in the two regions. The magnetic field was purely axial in the external plasma. Terradas \& Goossens (2012) studied standing kink waves in a magnetic tube with the magnetic twist confined in an annulus inside the tube.

In the magnetic tube considered in Paper I, the magnetic field is discontinuous at the tube boundary, which means that there is a surface current at this boundary. The magnetic field is also discontinuous at the annulus boundaries in the equilibrium considered by Karami \& Bahari (2012), so there are also surface currents at these boundaries. In this paper we aim to study kink oscillation in a magnetic tube with a more realistic equilibrium magnetic field that is continuous at the tube boundary. We only consider standing waves. Propagating kink waves in the same magnetic plasma configuration have been studied by Ruderman (2015).

The paper is organized as follows. In the next section we describe the equilibrium state and present the governing equations. In Sect. 3 we derive the integral equation describing the eigenfrequencies and eigenfunctions of standing waves. In Sect. 4 we calculate the eigenfrequencies of standing waves. In Sect. 5 we study the polarization of eigenfunctions. Section 6 contains the summary of the obtained results and our conclusions.

\section{Equilibrium state and governing equations}

We consider a twisted magnetic tube of radius $a$ and length $L$ homogeneous in the axial direction. The equilibrium plasma density is assumed to be constant inside and outside the tube. Hence, in cylindrical coordinates $r, \varphi, z$ with the $z$-axis coinciding with the tube axis, it is given by

$\rho= \begin{cases}\rho_{i}, & r<a, \\ \rho_{e}, & r>a,\end{cases}$

where $\rho_{i}$ and $\rho_{e}$ are constant. In what follows we use the subscripts " $i$ " and " $e$ " to distinguish between quantities inside and outside the tube. However, we drop these indices when it cannot cause confusion.

The equilibrium magnetic field only depends on $r$ and has two components, axial, $B_{z}$, and azimuthal, $B_{\varphi}$. In what follows we use the cold plasma approximation, so the equilibrium magnetic field must be force free. This condition reduces to

$\frac{\mathrm{d} B^{2}}{\mathrm{~d} r}=-\frac{2 B_{\varphi}^{2}}{r}$,

where $B^{2}=B_{z}^{2}+B_{\varphi}^{2}$. We assume that $B_{z e}$ is constant, $B_{\varphi i}$ is proportional to $r, B_{\varphi e}$ is inversely proportional to $r$, and the magnetic field is continuous at $r=a$. Then, using Eq. (2), we obtain that the equilibrium magnetic field is given by

$B_{z}^{2}=\left\{\begin{array}{cc}B_{0}^{2}+2 A^{2}\left(a^{2}-r^{2}\right), & r<a, \\ B_{0}^{2}, & r>a,\end{array} \quad B_{\varphi}= \begin{cases}A r, & r<a . \\ a^{2} A / r, & r>a .\end{cases}\right.$

where $A$ and $B_{0}$ are positive constants, and we assume that $B_{z}>0$.
The plasma motion is described by the linearized ideal MHD equations for cold plasmas,

$$
\begin{aligned}
& \rho \frac{\partial^{2} \boldsymbol{\xi}}{\partial t^{2}}=\frac{1}{\mu_{0}}(\nabla \times \boldsymbol{b}) \times \boldsymbol{B}+\frac{1}{\mu_{0}}(\nabla \times \boldsymbol{B}) \times \boldsymbol{b}, \\
& \boldsymbol{b}=\nabla \times(\boldsymbol{\xi} \times \boldsymbol{B}) .
\end{aligned}
$$

Here $\boldsymbol{\xi}=\left(\xi_{r}, \xi_{\varphi}, \xi_{z}\right)$ is the plasma displacement related to the plasma velocity $\boldsymbol{u}$ by $\boldsymbol{u}=\partial \boldsymbol{\xi} / \partial t, \boldsymbol{b}=\left(b_{r}, b_{\varphi}, b_{z}\right)$ the magnetic field perturbation, $\boldsymbol{B}$ the background magnetic field, $\rho$ the background plasma density, and $\mu_{0}$ the magnetic permeability of free space.

Ruderman (2007) has shown that Eqs. (4) and (5) can be transformed to

$$
\begin{aligned}
& \rho \frac{\partial^{2} \xi_{r}}{\partial t^{2}}=-\frac{\partial P}{\partial r}+\frac{B}{\mu_{0}} \mathcal{D}_{\|} b_{r}-\frac{2 B_{\varphi} b_{\varphi}}{r \mu_{0}} \\
& \rho \frac{\partial^{2} \xi_{\perp}}{\partial t^{2}}=-\mathcal{D}_{\perp} P+\frac{B}{\mu_{0}} \mathcal{D}_{\|} b_{\perp}+\frac{b_{r}}{\mu_{0}}\left[\frac{B_{z}}{r B} \frac{\mathrm{d}\left(r B_{\varphi}\right)}{\mathrm{d} r}-\frac{B_{\varphi}}{B} \frac{\mathrm{d} B_{z}}{\mathrm{~d} r}\right], \\
& b_{r}=B \mathcal{D}_{\|} \xi_{r}, \\
& b_{\perp}=B \mathcal{D}_{\|} \xi_{\perp}+\xi_{r}\left[\frac{B_{\varphi}}{r B} \frac{\mathrm{d}\left(r B_{z}\right)}{\mathrm{d} r}-\frac{B_{z}}{B} \frac{\mathrm{d} B_{\varphi}}{\mathrm{d} r}\right] \\
& P=-\frac{B_{\varphi}}{\mu_{0}} \frac{\partial\left(\xi_{r} B_{\varphi}\right)}{\partial r}-\frac{B_{z}}{r \mu_{0}} \frac{\partial\left(r \xi_{r} B_{z}\right)}{\partial r}-\frac{B^{2}}{\mu_{0}} \mathcal{D}_{\perp} \xi_{\perp}
\end{aligned}
$$

Here $P=\left(B_{\varphi} b_{\varphi}+B_{z} b_{z}\right) / \mu_{0}$ is the magnetic pressure perturbation, $\xi_{\perp}=\left(B_{z} \xi_{\varphi}-B_{\varphi} \xi_{z}\right) / B$, and $b_{\perp}=\left(B_{z} b_{\varphi}-B_{\varphi} b_{z}\right) / B$. The operators $\mathcal{D}_{\perp}$ and $\mathcal{D}_{\|}$are determined by

$\mathcal{D}_{\perp}=\frac{B_{z}}{r B} \frac{\partial}{\partial \varphi}-\frac{B_{\varphi}}{B} \frac{\partial}{\partial z}, \quad \mathcal{D}_{\|}=\frac{B_{\varphi}}{r B} \frac{\partial}{\partial \varphi}+\frac{B_{z}}{B} \frac{\partial}{\partial z}$.

We note that the component of the plasma displacement that is parallel to the equilibrium magnetic field, $\xi_{\|}=\left(B_{\varphi} \xi_{\varphi}+B_{z} \xi_{z}\right) / B$, is equal to zero in the cold plasma approximation.

Equations (6)-(10) have to be supplemented with the kinematic and dynamic boundary conditions at the tube boundary. The kinematic boundary condition states that the radial plasma displacement has to be continuous,

$\xi_{r i}=\xi_{r e} \quad$ at $\quad r=a$.

Because both the plasma displacement in the radial direction and the azimuthal magnetic field are continuous at the tube boundary, the dynamic boundary condition reduces to

$P_{i}=P_{e} \quad$ at $\quad r=a$.

The magnetic field lines are assumed to be frozen in the dense photospheric plasma. This means that the plasma displacement satisfies the boundary conditions

$\xi_{r}=0, \quad \xi_{\perp}=0 \quad$ at $\quad z=0, L$.

Finally, all perturbations have to vanish as $r \rightarrow \infty$. Equations (6)-(10) together with the boundary conditions (12)-(14) are used in the next section to derive the integral equation governing kink oscillations of the magnetic tube.

\section{Derivation of equation for radial displacement}

In this section we derive the governing equation for standing kink waves. Since we only consider kink oscillations, we take all variables proportional to $\mathrm{e}^{\mathrm{i} m \varphi}$, where $m= \pm 1$. In what follows we assume that the tube is thin, $a / L \ll \epsilon$. In accordance with this 
we introduce the scaled variable in the $z$-direction, $Z=\epsilon z$. The unscaled variable is $r$. The characteristic scale of variation in the radial direction is $a$, so the characteristic time corresponding to the radial direction is the Alfvénic time is $a / V_{A}$. On the other hand, the oscillation period is of the order of $L / V_{A}=\epsilon^{-1} a / V_{A}$, and it is also the characteristic time in the direction of the scaled variables $z$. In accordance with this we introduce the scaled time $T=\epsilon t$. To satisfy the Shafranov-Kruskal stability criterion (e.g. Roberts 1956; Shafranov 1957; Kruskal \& Tuck 1958), similar to Paper I, we assume that $A=\epsilon \widetilde{A}$ with $\widetilde{A} \lesssim B_{0} / a$. This assumption also agrees well with observations showing that the azimuthal component of magnetic field in coronal magnetic loops is much smaller than the axial component. We only consider eigenmodes of the kink oscillations. Hence we take perturbations of all variables proportional to $\exp (-\mathrm{i} \Omega T)$.

Now, eliminating $b_{r}$ and $b_{\perp}$ from Eqs. (6)-(10) we reduce this system of equations to equations for $\xi_{r}, \xi_{\perp}$ and $P$ :

$$
\begin{aligned}
& \left(\Omega^{2}-\mathcal{W}^{2}\right) \xi_{r}+\frac{2 \widetilde{B}_{\varphi}}{r \mu_{0} \rho} \frac{\partial\left(\widetilde{B}_{\varphi} \xi_{r}\right)}{\partial r}=\frac{\epsilon^{-2}}{\rho} \frac{\partial P}{\partial r}+\frac{2 B \widetilde{B}_{\varphi}}{r \mu_{0} \rho} \frac{\partial \xi_{\perp}}{\partial Z}+\mathcal{O}\left(\epsilon^{2}\right) \\
& \left(\Omega^{2}-\mathcal{W}^{2}\right) \xi_{\perp}=\frac{\mathrm{i} \epsilon^{-2}}{\rho} \mathcal{F} P-\frac{2 \mathrm{i} \widetilde{B}_{\varphi}}{r \sqrt{\mu_{0} \rho}} \mathcal{W} \xi_{r}+\mathcal{O}\left(\epsilon^{2}\right) \\
& P=-\frac{B^{2}}{r \mu_{0}} \frac{\partial\left(r \xi_{r}\right)}{\partial r}+\frac{2 \epsilon^{2} \widetilde{B}_{\varphi}^{2}}{r \mu_{0}} \xi_{r}-\frac{\mathrm{i} B^{2}}{\mu_{0}} \mathcal{F} \xi_{\perp}
\end{aligned}
$$

Here $\widetilde{B}_{\varphi}=\epsilon^{-1} B_{\varphi}$

$\mathcal{W}=\frac{1}{\sqrt{\mu_{0} \rho}}\left(\frac{m \widetilde{B}_{\varphi}}{r}-\mathrm{i} B_{0} \frac{\partial}{\partial Z}\right), \quad \mathcal{F}=\frac{m B_{z}}{r B}+\frac{\mathrm{i} \epsilon^{2} \widetilde{B}_{\varphi}}{B_{0}} \frac{\partial}{\partial Z}$.

Substituting the expression for $P$ given by Eq. (17) in Eq. (16) we obtain

$\mathcal{F}\left[\mathcal{F} \xi_{\perp}-\frac{i}{r} \frac{\partial\left(r \xi_{r}\right)}{\partial r}\right]=\epsilon^{2} \frac{\mu_{0} \rho}{B_{0}^{2}}\left(\Omega^{2}-\mathcal{W}^{2}\right) \xi_{\perp}+\epsilon^{2} \frac{2 \widetilde{B}_{\varphi}}{r B_{0}} \frac{\partial \xi_{r}}{\partial Z}+\mathcal{O}\left(\epsilon^{4}\right)$.

We consider this equation as an equation for $\xi_{\perp}$ and look for the solution in the form of the series expansion with respect to $\epsilon^{2}$,

$\xi_{\perp}=\xi_{\perp}^{(0)}+\epsilon^{2} \xi_{\perp}^{(1)}+\ldots$

After calculating the solution to Eq. (19) we substitute it in Eq. (17) to obtain the expression for $P$ in terms of $\xi_{r}$. Substituting this expression and expression for $\xi_{\perp}$ in Eq. (15) we obtain the equation for $\xi_{r}$. Then we express $P$ in terms of $\xi_{r}$. We carry out this procedure separately in the internal $(r<a)$ and external $(r>a)$ region.

\subsection{Solution inside the tube}

Using Eqs. (1) and (3) we write Eqs. (15), (17) and (19) in the internal region as

$$
\begin{gathered}
\left(\Omega^{2}-\mathcal{W}_{i}^{2}\right) \xi_{r}+\frac{2 \widetilde{A^{2}}}{\mu_{0} \rho_{i}} \frac{\partial\left(r \xi_{r}\right)}{\partial r}=\frac{\epsilon^{-2}}{\rho_{i}} \frac{\mathrm{d} P}{\mathrm{~d} r}+\frac{2 B_{0} \widetilde{A}}{\mu_{0} \rho_{i}} \frac{\partial \xi_{\perp}}{\partial Z}+\mathcal{O}\left(\epsilon^{2}\right) \\
P=-\frac{B_{i}^{2}}{r \mu_{0}} \frac{\partial\left(r \xi_{r}\right)}{\partial r}+\frac{2 \epsilon^{2} r \widetilde{A}^{2}}{\mu_{0}} \xi_{r}-\frac{i B_{i}^{2}}{\mu_{0}} \mathcal{F}_{i} \xi_{\perp}+\mathcal{O}\left(\epsilon^{4}\right) \\
\mathcal{F}_{i}\left[\mathcal{F}_{i} \xi_{\perp}-\frac{i}{r} \frac{\partial\left(r \xi_{r}\right)}{\partial r}\right]=\epsilon^{2} \frac{\mu_{0} \rho_{i}}{B_{0}^{2}}\left(\Omega^{2}-\mathcal{W}_{i}^{2}\right) \xi_{\perp} \\
+\epsilon^{2} \frac{2 \widetilde{A}}{B_{0}} \frac{\partial \xi_{r}}{\partial Z}+\mathcal{O}\left(\epsilon^{4}\right)
\end{gathered}
$$

where

$$
\mathcal{W}_{i}=\frac{1}{\sqrt{\mu_{0} \rho_{i}}}\left(m \widetilde{A}-\mathrm{i} B_{0} \frac{\partial}{\partial Z}\right), \quad \mathcal{F}_{i}=\frac{m B_{z i}}{r B_{i}}+\frac{\mathrm{i} \epsilon^{2} r \widetilde{A}}{B_{0}} \frac{\partial}{\partial Z} .
$$

In what follows we also use the expansions

$$
\begin{aligned}
& B_{z i}=B_{0}+\epsilon^{2} \widetilde{A}^{2} \frac{a^{2}-r^{2}}{B_{0}}+\mathcal{O}\left(\epsilon^{4}\right), \\
& B_{i}=B_{0}+\epsilon^{2} \widetilde{A}^{2} \frac{2 a^{2}-r^{2}}{2 B_{0}}+\mathcal{O}\left(\epsilon^{4}\right) .
\end{aligned}
$$

In the first-order approximation we collect terms of the order of unity in Eq. (23). As a result we obtain

$\xi_{\perp}^{(0)}=\frac{i}{m} \frac{\mathrm{d}\left(r \xi_{r}\right)}{\mathrm{d} r}$.

In the next-order approximation, we collect terms of the order of $\epsilon^{2}$ in Eq. (23). Then, using Eqs. (24)-(26) we obtain

$$
\begin{aligned}
\xi_{\perp}^{(1)}= & i m r^{2}\left(\frac{\partial^{2}}{\partial Z^{2}}+\frac{i m \widetilde{A}}{B_{0}} \frac{\partial}{\partial Z}\right. \\
& \left.+\frac{2 \mu_{0} \rho_{i} \Omega^{2}-\widetilde{A}^{2}}{2 B_{0}^{2}}\right) \frac{\partial\left(r \xi_{r}\right)}{\partial r}+\frac{2 r^{2} \widetilde{A}}{B_{0}} \frac{\partial \xi_{r}}{\partial Z} .
\end{aligned}
$$

Substituting Eqs. (26) and (27) in Eq. (22) and using Eq. (24) yields

$\epsilon^{-2} P=r \rho_{i}\left[\left(\Omega^{2}-\mathcal{W}_{i}^{2}\right) \frac{\partial\left(r \xi_{r}\right)}{\partial r}+\frac{2 m \widetilde{A}}{\sqrt{\mu_{0} \rho_{i}}} \mathcal{W}_{i} \xi_{r}\right]+\mathcal{O}\left(\epsilon^{2}\right)$.

Substituting Eqs. (26) and (28) in Eq. (21) and collecting terms of the order of unity in the obtained equation we find the equation for $\xi_{r}$ valid in the leading-order approximation with respect to $\epsilon^{2}$ :

$\left(\Omega^{2}-\mathcal{W}_{i}^{2}\right)\left(r^{2} \frac{\partial^{2} \xi_{r}}{\partial r^{2}}+3 r \frac{\partial \xi_{r}}{\partial r}\right)=0$.

Equation $\left(\Omega^{2}-\mathcal{W}_{i}^{2}\right) f=0$ describes Alfvén waves inside the tube. In what follows we eliminate these waves from the analysis. This implies that the expression in the second brackets in Eq. (29) is zero. Then the solution to Eq. (29) regular at $r=0$ is

$\xi_{r}=\eta(Z)$

where $\eta(Z)$ is an arbitrary function satisfying $\eta(0)=\eta(\ell)=0$, where $\ell=\epsilon L$. Substituting this result in Eq. (28) we obtain

$P=\frac{\epsilon^{2} r}{\mu_{0}}\left[B_{0}^{2} \frac{\mathrm{d}^{2} \eta}{\mathrm{d} Z^{2}}+\left(\mu_{0} \rho_{i} \Omega^{2}+\widetilde{A}^{2}\right) \eta\right]$.

\subsection{Equations in external region}

While the characteristic scale in the radial direction in the tube is $a$, this is, in general, not true outside the tube. In the whole external region this characteristic scale is L. However, the external region can be divided into two parts: The inner external region defined by the condition $r \sim a$, and the outer external region defined by the condition $r \gg a$. The characteristic scale in the radial direction in the inner external region is still equal to $a$, so we can use Eqs. (15)-(17) to describe the plasma motion in this region. In the outer external region we have to introduce the new scaled variable $R=\epsilon r$. Then we can obtain the solution in 
the external region using the method of matched asymptotic expansions. In accordance with this method we obtain the solution in the inner and outer external region separately, and then match the two solutions in the overlap region.

The solution in the outer external region is not used for the derivation of the governing integral equation. All that we need from this solution are the boundary conditions for the solution in the inner external region that are imposed by the matching conditions. In accordance with the matching conditions the asymptotic expression for the solution in the inner external region valid for large $r$ has to coincide with the asymptotic expression for the solution in the outer external region valid for small $R$. Since $B_{\varphi}$ is inversely proportional to $r$, it can be neglected in the outer external region and the magnetic field in this region can be considered as approximately homogeneous and in the $z$-direction. The solution describing the plasma motion in the presence of such an equilibrium magnetic field was obtained in Paper I. In this solution $P \propto R^{-1}$ and $\xi_{r} \propto R^{-2}$ for small $R$. Then the matching conditions imply that the asymptotic behaviour of the solution in the inner external region for large $r$ has to be $P \propto r^{-1}$ and $\xi_{r} \propto r^{-2}$. In accordance with this, in what follows, we look for the solution in the inner external region that vanishes as $r \rightarrow \infty$. Using Eqs. (1) and (3) we write Eqs. (15), (17) and (19) in the external region as

$$
\begin{aligned}
\left(\Omega^{2}-\mathcal{W}_{e}^{2}\right) \xi_{r}+\frac{2 a^{4} \widetilde{A}^{2}}{\mu_{0} \rho_{e} r^{2}} \frac{\partial}{\partial r}\left(\frac{\xi_{r}}{r}\right)= & \\
& \frac{\epsilon^{-2}}{\rho_{e}} \frac{\partial P}{\partial r}+\frac{2 a^{2} B_{0} \widetilde{A}}{\mu_{0} \rho_{e} r^{2}} \frac{\partial \xi_{\perp}}{\partial Z}+\mathcal{O}\left(\epsilon^{2}\right), \\
P= & -\frac{B_{e}^{2}}{r \mu_{0}} \frac{\partial\left(r \xi_{r}\right)}{\partial r}+\frac{2 \epsilon^{2} a^{4} \widetilde{A}^{2}}{\mu_{0} r^{3}} \xi_{r}-\frac{\mathrm{i} B_{e}^{2}}{\mu_{0}} \mathcal{F}_{e} \xi_{\perp}, \\
& \mathcal{F}_{e}\left[\mathcal{F}_{e} \xi_{\perp}-\frac{i}{r} \frac{\partial\left(r \xi_{r}\right)}{\partial r}\right]=\epsilon^{2} \frac{\mu_{0} \rho_{e}}{B_{0}^{2}}\left(\Omega^{2}-\mathcal{W}_{e}^{2}\right) \xi_{\perp} \\
& +\epsilon^{2} \frac{2 a^{2} \widetilde{A}}{r^{2} B_{0}} \frac{\partial \xi_{r}}{\partial r}+\mathcal{O}\left(\epsilon^{4}\right) .
\end{aligned}
$$

where

$\mathcal{W}_{e}=\frac{1}{\sqrt{\mu_{0} \rho_{e}}}\left(\frac{m a^{2} \widetilde{A}}{r^{2}}-\mathrm{i} B_{0} \frac{\partial}{\partial Z}\right), \quad \mathcal{F}_{e}=\frac{m B_{0}}{r B_{e}}+\frac{\mathrm{i} \epsilon^{2} a^{2} \widetilde{A}}{r B_{0}} \frac{\partial}{\partial Z}$.

In what follows we also use the expansion

$B_{e}=B_{0}+\epsilon^{2} \frac{a^{4} \widetilde{A^{2}}}{2 r^{2} B_{0}}+\mathcal{O}\left(\epsilon^{4}\right)$.

In the first-order approximation, the solution to Eq. (34) is again given by Eq. (26). In the next-order approximation, we obtain

$$
\begin{aligned}
\xi_{\perp}^{(1)}= & \frac{i}{m}\left(r^{2} \frac{\partial^{2}}{\partial Z^{2}}+\frac{\mathrm{i} a^{2} \widetilde{A}}{m B_{0}} \frac{\partial}{\partial Z}\right. \\
& \left.+\frac{\mu_{0} \rho_{e} r^{2} \Omega^{2}}{B_{0}^{2}}-\frac{a^{4} \widetilde{A^{2}}}{2 r^{2} B_{0}^{2}}\right) \frac{\partial\left(r \xi_{r}\right)}{\partial r}+\frac{2 a^{2} \widetilde{A}}{B_{0}} \frac{\partial \xi_{r}}{\partial Z} .
\end{aligned}
$$

Substituting Eqs. (35)-(37) in Eq. (33) yields

$$
\epsilon^{-2} P=r \rho_{e}\left[\left(\Omega^{2}-\mathcal{W}_{e}^{2}\right) \frac{\partial\left(r \xi_{r}\right)}{\partial r}+\frac{2 m a^{2} \widetilde{A}}{r^{2} \sqrt{\mu_{0} \rho_{e}}} \mathcal{W}_{e} \xi_{r}\right]+\mathcal{O}\left(\epsilon^{2}\right)
$$

Collecting terms of the order of unity in Eq. (32) and using Eqs. (26) and (38) we obtain, after some algebra, the equation for $\xi_{r}$ valid in the leading-order approximation with respect to $\epsilon^{2}$ :

$\frac{\partial}{\partial r}\left[r^{3}\left(\Omega^{2}-\mathcal{W}_{e}^{2}\right) \frac{\partial \xi_{r}}{\partial r}\right]=0$,

which implies that the expression in the square brackets is equal to an arbitrary function of $Z$, which we write in the form $B_{0}^{2} \chi(Z) /\left(\mu_{0} \rho_{e}\right)$. Then Eq. (39) reduces to

$\frac{\partial^{2} \psi}{\partial Z^{2}}+\frac{2 \mathrm{i} m \alpha}{r^{2}} \frac{\partial \psi}{\partial Z}+\left(\beta^{2}-\frac{\alpha^{2}}{r^{4}}\right) \psi=\frac{\chi(Z)}{r^{3}}$

where $\psi=\partial \xi_{r} / \partial r$ and

$\alpha=\frac{a^{2} \widetilde{A}}{B_{0}}, \quad \beta=\frac{\Omega \sqrt{\mu_{0} \rho_{e}}}{B_{0}}$.

It follows from Eq. (14) that $\psi$ satisfies the boundary conditions $\psi(0)=\psi(\ell)=0$

Since Eq. (40) does not contain the derivatives with respect to $r$, it can be considered as an ordinary differential equation for $\psi$ considered as a function of $Z$. Its solution satisfying the boundary conditions (42) is given by

$\psi=-\frac{1}{\beta r^{3}} \int_{0}^{\ell} \exp \left[\operatorname{im} \alpha r^{-2}(u-Z)\right] F(Z, u) \chi(u) \mathrm{d} u$,

where

$$
\begin{aligned}
F(Z, u)= & \frac{1}{\sin (\beta \ell)}\{H(Z-u) \sin (\beta u) \sin [\beta(\ell-Z)] \\
& +H(u-Z) \sin (\beta Z) \sin [\beta(\ell-u)]\},
\end{aligned}
$$

and $H$ is the Heaviside function. We note that this solution is only valid when $\beta \ell \neq \pi n, n=1,2, \ldots$ When $\beta \ell=\pi n$ the homogeneous counterpart of Eq. (40) has a non-trivial solution satisfying the boundary Eqs. (42). As a result a solution to Eq. (40) satisfying the boundary conditions (42) only exists when its right-hand side is orthogonal to the solution of its homogeneous counterpart. In what follows we eliminate $\beta \ell=\pi n$ from the analysis. To clarify the physical meaning of this condition we consider Alfvén oscillations outside the tube. They are described by the boundary value problem

$\left(\Omega^{2}-\mathcal{W}_{e}^{2}\right) \xi_{\perp}=0, \quad \xi_{\perp}(0)=\xi_{\perp}(\ell)=0$.

The solutions to this boundary value problem are

$\xi_{\perp}=\exp \left(-\frac{\mathrm{i} m \alpha Z}{r^{2}}\right) \sin \frac{\pi n Z}{\ell}, \quad \beta=\frac{\pi n}{\ell}$,

where $n=1$ corresponds to the fundamental mode and $n>1$ to the overtones. Hence, the condition $\beta \ell \neq \pi n$ means that we eliminate kink oscillations that are in resonance with the Alfvén oscillations outside the tube.

Integrating Eq. (43) with respect to $r$ and taking into account that $\xi_{r} \rightarrow 0$ as $r \rightarrow \infty$ we obtain

$\xi_{r}=\frac{\mathrm{i} m}{2 \alpha \beta} \int_{0}^{\ell} \frac{1-\exp \left[\mathrm{i} m \alpha r^{-2}(u-Z)\right]}{u-Z} F(Z, u) \chi(u) \mathrm{d} u$.

We note that $\xi_{r} \sim 1 / r^{2}$ when $r \gg a$, in complete agreement with the matching conditions at large $r$. Using Eqs. (35) and (39) we obtain from Eq. (38) in the leading-order approximation with respect to $\epsilon^{2}$ that

$P=\frac{\epsilon^{2} r}{\mu_{0}}\left[B_{0}^{2} \frac{\partial^{2} \xi_{r}}{\partial Z^{2}}+\left(\mu_{0} \rho_{e} \Omega^{2}+\frac{a^{4} \widetilde{A^{2}}}{r^{4}}\right) \xi_{r}\right]+\frac{\epsilon^{2} \chi B_{0}^{2}}{r \mu_{0}}$.

In particular, it follows from this expression that $P \sim 1 / r$ for $r \gg$ $a$, which is again in the complete agreement with the matching conditions at large $r$. 


\subsection{Matching solutions}

It follows from the boundary condition (12) that $\xi_{r}=\eta$ at $r=$ a. Using this result and substituting Eqs. (31) and (48) in the boundary condition (13), we obtain

$\chi=\left(\rho_{i}-\rho_{e}\right) \frac{\mu_{0} a^{2} \Omega^{2}}{B_{0}^{2}} \eta$.

Substituting this result and $\xi_{r}=\eta$ in Eq. (47), and returning to the original non-scaled variable $z$, we obtain the equation determining $\eta$ :

$$
\begin{aligned}
\eta(z)= & \frac{i m \omega\left(\rho_{i}-\rho_{e}\right) \sqrt{\mu_{0}}}{2 A \sqrt{\rho_{e}}} \\
& \times \int_{0}^{L} \frac{1-\exp \left[\operatorname{im} A(u-z) / B_{0}\right]}{u-z} F(z, u) \eta(u) \mathrm{d} u,
\end{aligned}
$$

where $\omega=\epsilon \Omega, \omega$ is substituted for $\Omega$ in the expression for $\beta$, and $L$ is substituted for $\ell$ in the expression for $F$.

\section{Eigenfrequencies of kink oscillations}

We introduce the Alfvén speed at large distance from the tube, $V=B_{0} / \sqrt{\mu_{0} \rho_{e}}$, and the dimensionless quantities

$\zeta=\frac{\rho_{i}}{\rho_{e}}, s=\frac{2 z-L}{L}, q=\frac{A L}{B_{0}}, h=\frac{\eta}{a} \exp \left(\frac{\mathrm{i} m q s}{4}\right), \varpi=\frac{\omega L}{V}$.

The quantity $q$ is especially important in our analysis. To clarify its physical meaning, we consider the equation of a magnetic field line inside the tube,

$$
\frac{\mathrm{d} \varphi}{A}=\frac{\mathrm{d} z}{B_{0}},
$$

where we have taken $B_{z} \approx B_{0}$. Integrating this equation we obtain

$\varphi=\frac{z A}{B_{0}}+$ const.

It follows from this equation that the $\varphi$-coordinate of a point on a magnetic field line varies by the angle $\Delta \varphi=q$ when this point moves along the field line from one footpoint to the other. The Kruskal-Shafranov stability criterion can be written as $q \lesssim 2 \pi$. In the dimensionless variables Eq. (50) is rewritten as

$h(s)=\frac{\varpi(\zeta-1)}{q \sin \varpi} \int_{-1}^{1} \frac{\sin [q(s-u) / 4]}{s-u} G(s, u) h(u) \mathrm{d} u$,

where

$$
\begin{aligned}
G(s, u)= & H(s-u) \sin [\varpi(1+u) / 2] \sin [\varpi(1-s) / 2] \\
& +H(u-s) \sin [\varpi(1+s) / 2] \sin [\varpi(1-u) / 2] .
\end{aligned}
$$

First of all, we note that Eq. (54) is independent of $m$. We also note that without loss of generality, we can assume that $h(s)$ is a real function. The right-hand side of Eq. (54) does not change sign when $\varpi$ changes sign. This implies that if $\varpi$ is an eigenvalue of the integral Eq. (50), then $-\varpi$ is also an eigenvalue, which completely agrees with the general spectral theory of ideal linear MHD (e.g. Goedbloed \& Poedts 2004). Changing the sign of $s$ and $u$ in Eq. (54) we obtain

$$
h(-s)=\frac{\varpi(\zeta-1)}{q \sin \varpi} \int_{-1}^{1} \frac{\sin [q(s-u) / 4]}{s-u} G(s, u) h(-u) \mathrm{d} u .
$$

It follows from Eqs. (54) and (56) that both $h(s)+h(-s)$ and $h(s)-h(-s)$ are solutions to Eq. (54). If $\varpi$ is an eigenvalue, then at least one of these two functions is not identically equal to zero. This means that we can only search for solutions to Eq. (54) that are either even or odd.

Now we assume that the twist is weak, $q \ll 1$, and use the regular perturbation method to obtain a solution to Eq. (54). In accordance with this method, we search for the solution in the form

$h=h_{0}+q^{2} h_{1}+\ldots, \quad \varpi=\varpi_{0}+q^{2} \varpi_{1}+\ldots$,

where we have taken into account that the expansion of the righthand side of Eq. (54) with respect to $q$ only contains even powers of $q$. The first terms in these expressions describe kink waves in an untwisted magnetic tube. Substituting these expressions in Eq. (54) we obtain in the zero order approximation

$\mathcal{L}\left[h_{0}\right] \equiv h_{0}-\frac{\varpi_{0}(\zeta-1)}{4 \sin \varpi_{0}} \int_{-1}^{1} G_{0}(s, u) h_{0}(u) \mathrm{d} u=0$,

where $G_{0}(s, u)$ is given by Eq. (55) with $\varpi_{0}$ substituted for $\varpi$. This is straightforward to verify by the direct substitution that

$h_{0}(s)=h_{0 f}=\cos \frac{\pi s}{2}, \quad \varpi_{0}=\varpi_{0 f}=\pi \sqrt{\frac{2}{\zeta+1}}$

satisfy Eq. (58). Since this is a linear problem, we can take the amplitude of $h_{0}(s)$ equal to unity without loss of generality. In the dimensional variables the expression for the frequency in the zero order approximation is

$\omega=C_{k} \frac{\pi}{L}, \quad C_{k}^{2}=\frac{2 B_{0}^{2}}{\mu_{0}\left(\rho_{i}+\rho_{e}\right)}$,

which corresponds to the fundamental mode of kink oscillations, in complete agreement with the general theory of kink oscillations (e.g., Ruderman \& Erdélyi 2009).

In the first-order approximation, after some algebra, we obtain from Eq. (54)

$$
\begin{aligned}
\mathcal{L}\left[h_{1}\right]= & \frac{\varpi_{1}(\zeta-1)}{4 \sin \varpi_{0}} \int_{-1}^{1}\left[\varpi_{0} G_{1}(s, u)\right. \\
& \left.+\left(1-\varpi_{0} \cot \varpi_{0}\right) G_{0}(s, u)\right] h_{0}(u) \mathrm{d} u \\
& -\frac{\varpi_{0}(\zeta-1)}{384 \sin \varpi_{0}} \int_{-1}^{1}(s-u)^{2} G_{0}(s, u) h_{0}(u) \mathrm{d} u
\end{aligned}
$$

where

$$
\begin{aligned}
G_{1}(s, u)= & H(s-u)\left(\frac{1+u}{2} \cos \frac{\varpi_{0}(1+u)}{2} \sin \frac{\varpi_{0}(1-s)}{2}\right. \\
& \left.+\frac{1-s}{2} \sin \frac{\varpi_{0}(1+u)}{2} \cos \frac{\varpi_{0}(1-s)}{2}\right) \\
& +H(u-s)\left(\frac{1+s}{2} \cos \frac{\varpi_{0}(1+s)}{2} \sin \frac{\varpi_{0}(1-u)}{2}\right. \\
& \left.+\frac{1-u}{2} \sin \frac{\varpi_{0}(1+s)}{2} \cos \frac{\varpi_{0}(1-u)}{2}\right)
\end{aligned}
$$

Since the equation $\mathcal{L}\left[h_{1}\right]=0$ has a non-trivial solution, Eq. (61) can only be solved if its right-hand side satisfies the compatibility condition. To obtain this condition we multiply Eq. (61) 


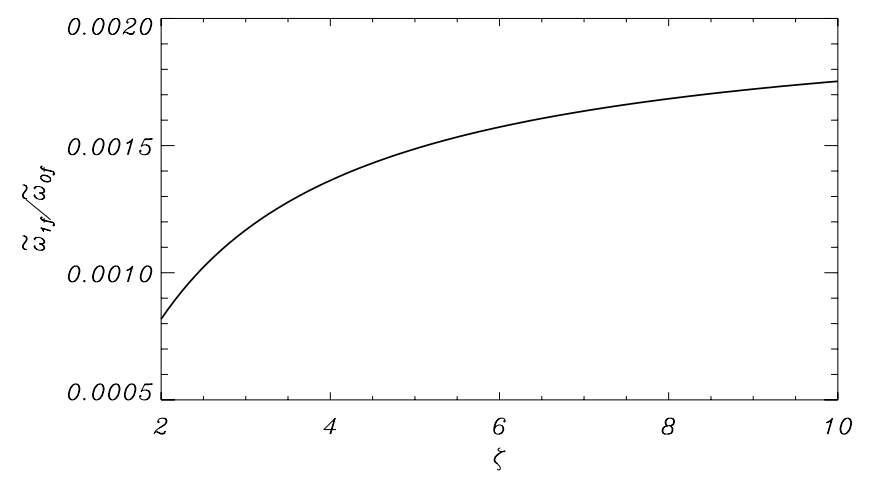

Fig. 1. Dependence of the relative correction to the fundamental frequency, $\varpi_{1 f} / \varpi_{0 f}$, on $\zeta$.

by $h_{0}(s)$ and integrate with respect to $s$ over the interval $[-1,1]$. Then, using the symmetry $G_{0}(s, u)=G_{0}(u, s)$, we obtain

$\int_{-1}^{1} h_{0}(s) \mathcal{L}\left[h_{1}\right] \mathrm{d} s=\int_{-1}^{1} h_{1}(s) \mathcal{L}\left[h_{0}\right] \mathrm{d} s=0$.

Hence, the compatibility condition is that the integral from the right-hand side of Eq. (61) multiplied by $h_{0}(s)$ and integrated with respect to $s$ over the interval $[-1,1]$ is zero. It follows from Eq. (58) that

$\frac{\zeta-1}{4 \sin \varpi_{0}} \int_{-1}^{1} h_{0}(s) \mathrm{d} s \int_{-1}^{1} G_{0}(s, u) h_{0}(u) \mathrm{d} u=\frac{1}{2 \varpi_{0}}$.

After long but straightforward calculation we obtain

$$
\begin{gathered}
\frac{\zeta-1}{4 \sin \varpi_{0}} \int_{-1}^{1} h_{0}(s) \mathrm{d} s \int_{-1}^{1} G_{1}(s, u) h_{0}(u) \mathrm{d} u= \\
\frac{\cot \varpi_{0}}{2 \varpi_{0}}+\frac{\zeta+3}{2 \varpi_{0}^{2}(\zeta-1)}, \\
\frac{\varpi_{0}(\zeta-1)}{384 \sin \varpi_{0}} \int_{-1}^{1} h_{0}(s) \mathrm{d} s \int_{-1}^{1}(s-u)^{2} G_{0}(s, u) h_{0}(u) \mathrm{d} u= \\
\frac{\varpi_{0}(\zeta+1)}{6 \pi^{2}(\zeta-1)^{2}}\left(\frac{8(\zeta+1)^{2}}{\pi^{2}(\zeta-1)} \cot \frac{\varpi_{0}}{2}-\frac{3 \zeta+5}{2 \varpi_{0}}\right) .
\end{gathered}
$$

Then, using the compatibility condition for Eq. (61), we obtain with the aid of Eqs. (64)-(66) that the correction to the funndamental frequency is given by

$\varpi_{1 f}=\frac{1}{6\left(\zeta^{2}-1\right)}\left(\frac{16(\zeta+1)^{2}}{\pi^{2}(\zeta-1)} \cot \frac{\varpi_{0 f}}{2}-\frac{3 \zeta+5}{\varpi_{0 f}}\right)$.

The dependence of $\varpi_{1 f} / \varpi_{0 f}$ on $\zeta$ is shown in Fig. 1.

Now we obtain the second solution corresponding to the first overtone. In the zero-order approximation we have

$h_{0}(s)=h_{0 \mathrm{o}}=\sin (\pi s), \quad \varpi_{0}=\varpi_{0 \mathrm{o}}=2 \pi \sqrt{\frac{2}{\zeta+1}}$.

Equations (63)-(65) remain valid, while instead of Eq. (66) we obtain equation

$$
\begin{gathered}
\frac{\varpi_{0}(\zeta-1)}{384 \sin \varpi_{0}} \int_{-1}^{1} h_{0}(s) \mathrm{d} s \int_{-1}^{1}(s-u)^{2} G_{0}(s, u) \eta_{0}(u) \mathrm{d} u= \\
-\frac{\varpi_{0}(\zeta+1)}{24 \pi^{2}(\zeta-1)^{2}}\left(\frac{2(\zeta+1)^{2}}{\pi^{2}(\zeta-1)} \tan \frac{\varpi_{0}}{2}+\frac{3 \zeta+5}{2 \varpi_{0}}\right) .
\end{gathered}
$$

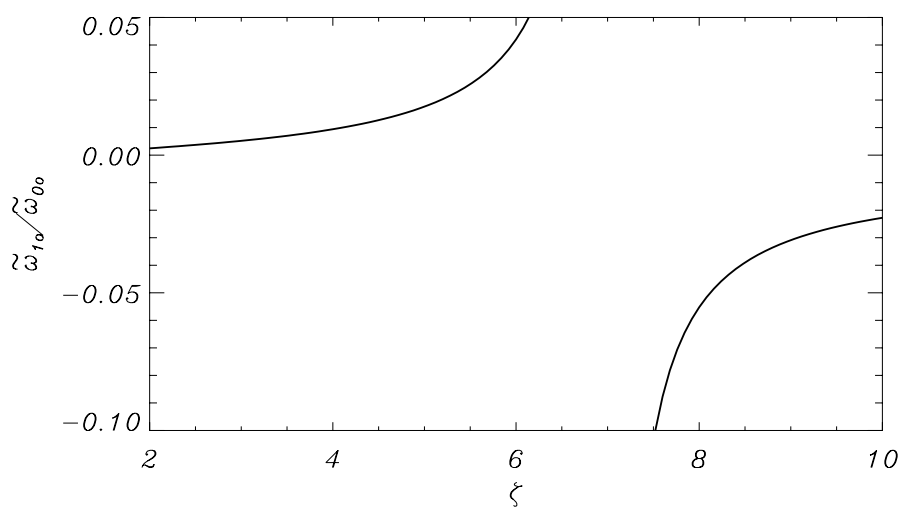

Fig. 2. Dependence of the relative correction to the first overtone, $\varpi_{1 \mathrm{o}} / \varpi_{0 \mathrm{o}}$, on $\zeta$.

Using these results we obtain that the correction to the firstovertone frequency is given by

$\varpi_{1 \mathrm{o}}=-\frac{1}{6\left(\zeta^{2}-1\right)}\left(\frac{4(\zeta+1)^{2}}{\pi^{2}(\zeta-1)} \tan \frac{\varpi_{0 \mathrm{o}}}{2}+\frac{3 \zeta+5}{\varpi_{0 \mathrm{o}}}\right)$.

The dependence of $\varpi_{10} / \varpi_{0 o}$ on $\zeta$ is shown in Fig. 2.

Note that the expression for $\varpi_{10}$ has a singularity when $\varpi_{0 \mathrm{o}}=\pi$, that is, when $\zeta=7$. This singularity is related to the fact that, at this value of $\zeta$, the frequency of the first overtone in the zero-order approximation with respect to $q$ coincides with the frequency of the fundamental mode of the Alfvén oscillations outside the tube, so the kink oscillation is in resonance with the Alfvén oscillation. The expression for $\varpi_{1 f}$ does not have any singularities because $\varpi_{0 f}$ is smaller than the frequency of the fundamental mode of the Alfvén oscillations outside the tube.

Here one needs to clarify one important point. In the case of propagating waves the Alfvén frequency for a given axial wave number is constant inside the tube, but it depends on the radial variable outside the tube. As a result, there is an Alfvén continuum and some of propagating wave modes are subject to resonant damping (Ruderman 2015). However, in the case of standing waves the situation is completely different. The eigenfrequency of Alfvén oscillations outside the tube is defined by the eigenvalue problem Eq. (45). It defines exactly one eigenfrequency of the fundamental mode and one eigenfrequecy for each overtone. Hence, there is no Alfvén continuum and, as a result, no wave damping due to resonant absorption.

It is worth noting that the resonance between the kink and Alfvén oscillation does not cause any problem when $q=0$, that is, in the case of an untwisted tube. The reason for this is the following. As we can see from Eq. (49), $\chi$ is proportional to $\eta$. When $\Omega$ coincides with the eigenfrequency of one of the Alfvén oscillations, Eq. (40) has a solution when its right-hand side is orthogonal to the corresponding Alfvén eigenmode. When $q=0$, it follows from Eq. (46) that the eigenfunction corresponding to the fundamental mode of the Alfvén oscillation is proportional to $\cos (\pi s / 2)$. The eigenfunction corresponding to the first overtone of kink oscillations is proportional to $\sin (\pi s)$, so it is obvious that the orthogonality condition is satisfied.

In contrast, the orthogonality condition is not satisfied when $q \neq 0$. This can be easily proved in the case of small $q$. In this case it follows from Eq. (46) that the eigenfunction 
M. S. Ruderman \& J. Terradas: Kink oscillations of twisted magnetic tubes

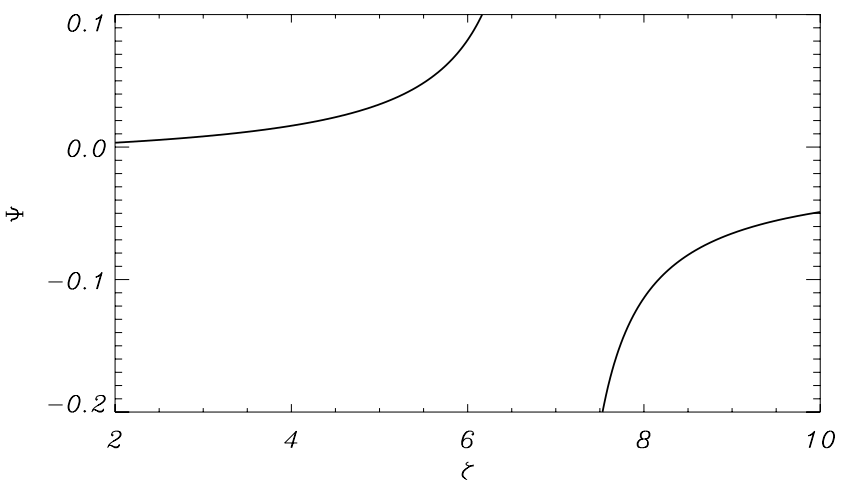

Fig. 3. Dependence of the correction to the frequency ratio, $\Psi$, defined by Eq. (72), on $\zeta$.

corresponding to the fundamental mode of the Alfvén oscillation is given by

$\exp \left(-\frac{\mathrm{i} m q a^{2}(s+1)}{2 r^{2}}\right) \cos \frac{\pi s}{2}=\left(1-\frac{\mathrm{i} m q a^{2}(s+1)}{2 r^{2}}\right) \cos \frac{\pi s}{2}+\mathcal{O}\left(q^{2}\right)$.

The eigenfunction corresponding to the first overtone of kink oscillations is

$\exp \left(\frac{\mathrm{i} m q s}{4}\right) \sin (\pi s)+\mathcal{O}\left(q^{2}\right)=\left(1+\frac{\mathrm{i} m q s}{4}\right) \sin (\pi s)+\mathcal{O}\left(q^{2}\right)$.

Then the condition that the right-hand side of Eq. (40) is orthogonal to the fundamental Alfvén eigenmode is written as

$\int_{-1}^{1} \sin (\pi s) \cos \frac{\pi s}{2}\left(\frac{s}{4}-\frac{a^{2}(s+1)}{2 r^{2}}\right) \mathrm{d} s=\mathcal{O}(q)$.

After integration this equation reduces to

$\frac{r^{2}-2 a^{2}}{r^{2}}=\mathcal{O}(q)$

It is obvious that this relation cannot be satisfied for all values of $r$.

The ratio of frequencies of the first overtone and fundamental mode is given by the expression

$\frac{\varpi_{\mathrm{o}}}{\varpi_{\mathrm{f}}}=2+q^{2} \Psi(\zeta)+\mathcal{O}\left(q^{4}\right)$

where

$$
\begin{aligned}
\Psi(\zeta)= & \frac{3 \zeta+5}{8 \pi^{2}(\zeta-1)}-\frac{(\zeta+1) \sqrt{2(\zeta+1)}}{3 \pi^{3}(\zeta-1)^{2}} \\
& \times\left(\tan \frac{\pi \sqrt{2}}{\sqrt{\zeta+1}}+8 \cot \frac{\pi \sqrt{2}}{2 \sqrt{\zeta+1}}\right) .
\end{aligned}
$$

Figure 3 shows the dependence of $\Psi$ on $\zeta$. Note that $\Psi$ also has singularity at $\zeta=7$. We see that the magnetic twist enhances the ratio $\varpi_{\mathrm{o}} / \varpi_{\mathrm{f}}$ when $\zeta<7$, and reduces this ratio when $\zeta>7$.

We also calculated the dependence of $\varpi$ on $q$ numerically for the fundamental mode and first overtone, and for three various values of $\zeta$. In this calculation we used the full system of linearized ideal MHD equations for cold plasmas (see equations and details about the method in Terradas \& Goossens 2012). The results are shown in Fig. 4. We can see that the frequency of the fundamental mode calculated analytically practically coincides with that calculated numerically for $q \lesssim 6$, where $q=2 \pi \approx 6.28$

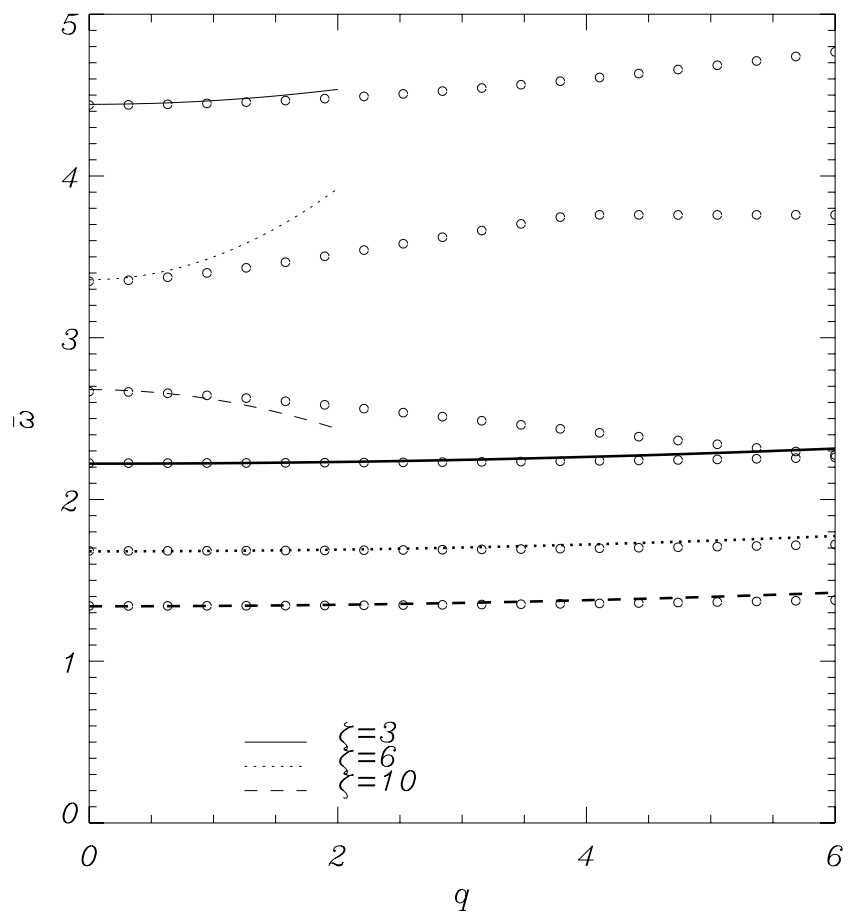

Fig. 4. Dependence of $\varpi_{\mathrm{f}}$ and $\varpi_{\mathrm{o}}$ on $q$. The lines show the approximated dependence valid for small $q$ and determined by Eqs. (59) and (67) for the fundamental mode, and by Eqs. (68) and (70) for the first overtone. The solid, dotted and dashed lines correspond to for $\zeta=3,6$, and 10. The lower three lines correspond to the fundamental mode, and the upper three lines to the overtones. The circles show the numerical results.

corresponds to the full turn of helical magnetic field lines inside the tube on the loop length. Moreover, the correction related to the twist is practically negligible.

The agreement between the analytical and numerical results is poorer for the frequency of the first overtone. However, even in this case, the analytical approximation works fairly well for $q \lesssim 2$ when $\zeta=3$ and $\zeta=10$, and only when $\zeta=6$ the analytical and numerical results only agree well for $q \lesssim 1$. This more severe restriction on the value of $q$ when $\zeta=6$ is related to the fact that this value is close to 7 . As we have seen, the fist overtone frequency has a singularity when $\zeta=7$. It follows from the numerical results that the correction related to the twist is fairly small for $q \lesssim 2 \pi$. Hence, we see that if the equilibrium magnetic field satisfies the Kruskal-Shafranov stability criterion, i.e. when the ratio of the azimuthal to axial magnetic field is lower than or of the order of $\epsilon=a / L$, the effect of twist on the frequencies of fundamental mode and first overtone is sufficiently small.

In Fig. 5 we show the dependence of the ratio of the first overtone frequency to the fundamental frequency, $\varpi_{\mathrm{o}} / \varpi_{\mathrm{f}}$. The numerical results confirm the conclusion based on the analytical results that the twist enhances the frequency ratio when $\zeta<7$ and reduces it when $\zeta>7$. Again the agreement between the analytical and numerical results strongly depends on the value of $\zeta$. When $\zeta=3$, the agreement is fairly good for $q \lesssim 2$. When $\zeta=10$, the analytical and numerical results agree fairly well for $q \lesssim 1$. Finally, When $\zeta=6$, the analytical and numerical results only agree for $q \ll 1$.

Up to now two properties of coronal loops that can affect the frequency ratio have been studied. The first property is the density variation along the loop related to the coronal stratification. This property causes the decrease in the frequency ratio. It was suggested by Andries et al. (2005) as a tool for estimating the 


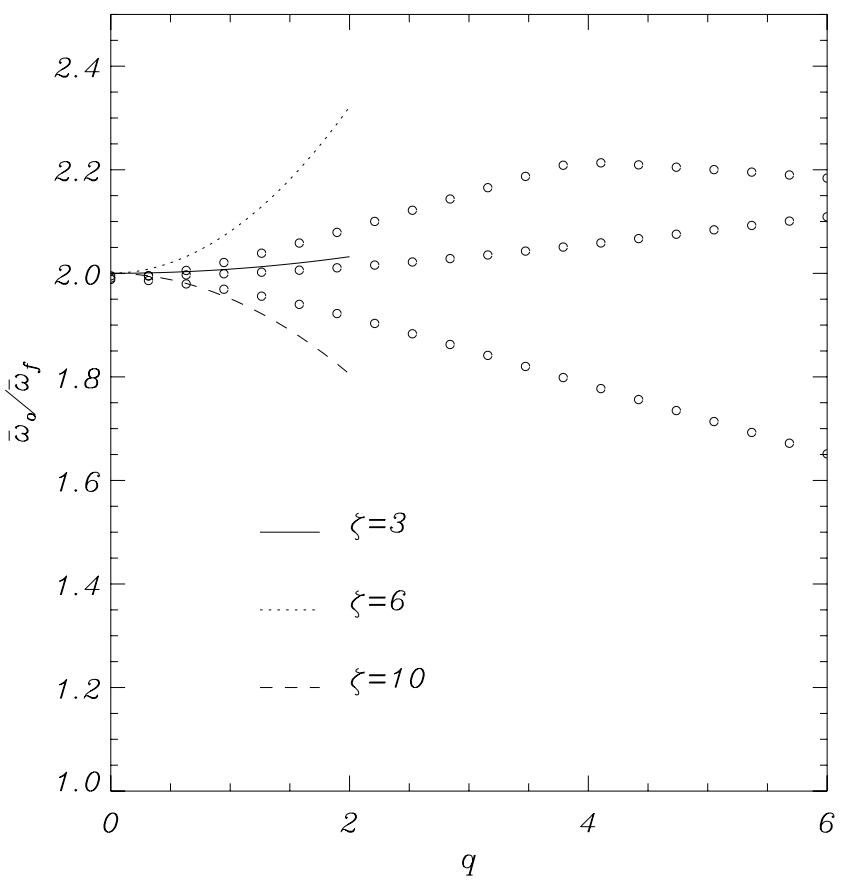

Fig. 5. Dependence of $\varpi_{\mathrm{o}} / \varpi_{\mathrm{f}}$ on $q$. The lines show the approximated dependence valid for small $q$ and determined by Eqs. (71) and (72). The solid, dotted and dashed lines correspond to for $\zeta=3,6$ and 10 . The circles show the numerical results.

density scale height in the corona using simultaneous observations of the fundamental harmonic and first overtone of coronal loop kink oscillations (see also the review by Andries et al. 2009). The second property is the coronal loop expansion. This property causes the increase in the frequency ratio (Ruderman et al. 2008; Verth \& Erdélyi 2008; Verth et al. 2008). Now we have a third property, the magnetic twist. It enhances the frequency ratio similarly to the loop expansion when the density contrast is moderate, and reduces this ratio similarly to the density variation when the density contrast is high. This result should be taken into account in coronal seismology.

\section{Wave polarization}

To study the wave mode polarization we need to obtain real solutions for $\xi_{r}$ and $\xi_{\varphi}$. We recall that without loss of generality, we can assume that solutions to Eq. (54) are real. Then, using the relation between $\eta$ and $h$ given by Eq. (51) and restoring the dependence on $t$ and $\varphi$, we obtain that inside the tube,

$\xi_{r}=a h(s) \exp [\mathrm{i}(m \varphi-m q s / 4-\omega t)]$.

Now it follows from Eq. (26) that

$\xi_{\varphi}=\mathrm{i} m a h(s) \exp [\mathrm{i}(m \varphi-m q s / 4-\omega t)]$,

where we have used the fact that the difference between $\xi_{\varphi}$ and $\xi_{\perp}$ is of the order of $\epsilon$ and thus can be neglected. Now we recall that if $\omega$ is an eigenfrequency and the corresponding solution to Eq. (54) is $h(s)$, then $-\omega$ is also an eigenfrequency and the corresponding solution to Eq. (54) is the same $h(s)$, and the eigenfrequency is independent of $m$. Then we take the superposition of two solutions, one given by Eqs. (73) and (74) with $m=1$, and the second given by the same equations but with $m=-1$ and with $-\omega$ substituted for $\omega$. As a result, we obtain

$\xi_{r}=a h(s) \cos (\omega t-\varphi+q s / 4)$,

$\xi_{\varphi}=a h(s) \sin (\omega t-\varphi+q s / 4)$.
We introduce the auxiliary Cartesian coordinates $x, y$ in the plane perpendicular to the tube axis with the angle $\varphi$ measured from the $x$-axis. Then

$\xi_{x}=\xi_{r} \cos \varphi-\xi_{\varphi} \sin \varphi=a h(s) \cos (\omega t+q s / 4)$,

$\xi_{y}=\xi_{r} \sin \varphi+\xi_{\varphi} \cos \varphi=a h(s) \sin (\omega t+q s / 4)$.

This solution describes a circularly polarized wave. At a fixed $a$, the displacement vector rotates in the couterclockwise direction, so the wave is right-hand polarized. Changing the sign of $\omega$, we obtain the second solution

$\xi_{x}=a h(s) \cos (\omega t-q s / 4), \quad \xi_{y}=-a h(s) \sin (\omega t-q s / 4)$.

This solution describes a circularly polarized wave with a lefthand polarization. Using the linear combination of the two circularly polarized waves, we can obtain a wave with arbitrary elliptic polarization. We are particularly interested in the wave with the linear polarization. We obtain the solution describing the linearly polarized wave taking the half-sum of the two solutions given by Eqs. (76) and (77):

$\xi_{x}=a h(s) \cos (\omega t) \cos (q s / 4)$,

$\xi_{y}=a h(s) \cos (\omega t) \sin (q s / 4)$.

We see that the polarization direction varies along the tube axis. It constitutes the angle $q s / 4$ with the positive $x$-direction measured counterclockwise. Note that the effect of variation of the polarization direction of linearly polarized kink oscillations along a twisted tube has previously been reported by Ruderman \& Scott (2011) and Terradas \& Goossens (2012). In particular, Eq. (78) agrees with Eqs. (60) and (61) of Terradas \& Goossens (2012) in the limit of very small twist.

All the results in this article have been obtained under the assumption that $A>0$. If $A<0$, all the results remain the same except for he direction of polarization. In particular, the direction of polarization of a linearly polarized oscillation would constitute an angle $q s / 4$ with the positive $x$-direction measured clockwise.

\section{Summary and conclusions}

We studied the kink oscillations of a twisted magnetic tube. We assumed that the tube is thin and the twist is weak, that is, the ratio of the azimuthal and axial component of the background magnetic field is of the order of the small parameter $\epsilon$ equal to the ratio of the tube radius to its length. We took the azimuthal component of the background magnetic field to be proportional to the distance $r$ from the tube axis inside the tube, and inversely proportional to $r$ outside the tube. The background magnetic field was continuous at the tube boundary. We also assumed that the plasma density is constant inside and outside the tube, and we used the cold plasma approximation.

Using the method of asymptotic expansions we derived the governing equation for kink oscillations valid in the leadingorder approximation with respect to $\epsilon$. It is a complicated integral equation that cannot be solved analytically in the general case. To make analytical progress we introduced the parameter $q$, which is the ratio of azimuthal to axial component of the magnetic field at the tube boundary divided by $\epsilon$. Then we assumed that $q \ll 1$ and found the solutions to the integral equation in the form of expansion with respect to $q^{2}$. We restricted our analysis to the fundamental mode and first overtone and only calculated the first two terms of this expansion. Hence, we obtained the 
corrections proportional to $q^{2}$ to the frequencies of the fundamental mode and first overtone of a straight magnetic tube. We studied the dependence of these correction of the ratio of densities inside and outside the tube, $\zeta$. In particular, we obtained that the correction to the first overtone has a singularity at $\zeta=7$. This singularity is related to the fact that the frequency of the first overtone coincides with the fundamental Alfvén frequency in the external plasma at this value of $\zeta$.

The analytical solution was compared to the numerical solution obtained using the full set of ideal linear MHD equations. Although the analytical expressions for the eigenmode frequencies were obtained under assumption that $q \ll 1$, the analytical expression for the fundamental mode frequency agrees very well with the numerical one for $q \leq 6$. The correction to the fundamental mode frequency related to the magnetic twist is very small.

The analytical expression for the first-overtone frequency agrees with that calculated numerically for $q \lesssim 1$ alone. The numerically calculated correction to the frequency of the fist overtone of a straight tube related to the magnetic twist remains moderate for $q \leq 6$.

We also calculated the ratio of frequencies of the first overtone and fundamental mode, which is especially important for coronal seismology. We found that the twist enhances this ratio when $\zeta<7$ and reduces it when $\zeta>7$. In general, when $q \simeq 6$, the difference between this ratio and 2 is similar to the values obtained in the simultaneous observations of the fundamental harmonic and first overtone of the coronal loop kink oscillations (Verwichte et al. 2004; van Doorsselaere et al. 2007, 2009; Andries et al. 2009). This result implies that the observed deviation of the frequency ratio from 2 can be related not only with the density variation along the loop and loop expansion, but also with the presence of magnetic twist.

Finally, we studied the eigenmode polarization. We found that, in general, the eigenmodes are elliptically polarized. In a particular case of linear polarization the polarization direction rotates along the loop.

Comparing the results obtained in this article to those obtained previously, we note that they are different from those presented in Paper I. We had shown in Paper I that the magnetic twist does not affect the period ratio of the fundamental harmonic and first overtone, while in this article we obtained that it does affect the period ratio. The latter result also agrees very well with those obtained by Karami \& Bahari (2012) and Terradas \& Goossens (2012). Hence, we can conclude that the result obtained in Paper I is only valid for the particular equilibrium considered in that article, while, in general, the magnetic twist affects the period ratio. It does not make very much sense to compare the results obtained in this article with those obtained by Karami \& Bahari (2012) and Terradas \& Goossens (2012) quantitatively because the equilibria considered in these articles are completely different from that used in this article.

To study the standing kink waves we have used a relatively simple model of a magnetic tube with the constant electrical current density inside the tube and current free environment. The assumption that the environment is current free, so that the current is only concentrated inside the tube, seems to be quite general. If it is satisfied then the equilibrium magnetic field outside the tube is independent of a particular distribution of the electrical current inside the tube. Its axial component is constant and the azimuthal component is inversely proportional to the distance from the tube axis. If the current density inside the tube varies in the radial direction then the local Alfvén frequency will also vary in the radial direction. Depending on a particular equilibrium this may or may not lead to wave damping due to resonant absorption. However, we can expect that other properties of the waves will remain qualitatively the same.

Acknowledgements. A part of this work was carried out when MSR was a guest of Departament de Físika of Universitat de les Illes Balears. He acknowledges the financial support received from the Universitat de les Illes Balears and the warm hospitality of the departament. He also acknowledges the support by the STFC grant. J.T. acknowledges support from the Spanish Ministerio de Educación y Ciencia through a Ramón y Cajal grant and funding provided under the project AYA2011-22846 by the Spanish MICINN and FEDER Funds.

\section{References}

Andries, J., Arregui, I., \& Goossens, M. 2005, ApJ, 624, L57

Andries, J., van Doorsselaere, T., Roberts, B., et al. 2009, Space Sci. Rev., 149, 3

Aschwanden, M. J., Fletcher, L., Schrijver, C. J., \& Alexander, D. 1999, ApJ 520,880

Ballai, I., \& Erdélyi, R. 2002, J. Plasma Phys., 67, 79

Bennett, K., Roberts, B., \& Narain, U. 1999, Sol. Phys., 185, 41

Bogdan, T. J. 1984, ApJ, 282, 769

Browning, P. K., \& Priest, E. R. 1983, ApJ, 266, 848

Carter, P. S., \& Erdélyi, R. 2007, A\&A, 475, 323

Carter, P. S., \& Erdélyi, R. 2008, A\&A, 481, 239

Dungey, J. W., \& Loughead, R. E. 1954, Austr. J. Phys., 7, 5

Edwin, P. M., \& Roberts, B. 1983, Sol. Phys., 88, 179

Erdélyi, R., \& Carter, B. K. 2006, A\&A, 455, 361

Erdélyi, R., \& Fedun, V. 2007, Science, 318, 1572

Goedbloed, J. P. H., \& Poedts, S. 2004, Principles of Magnetohydrodynamics (Cambridge, UK: Cambridge University Press)

Goossens, M., \& Ruderman, M. S. 1995, Phys. Scr., 60, 171

Goossens, M., Ruderman, M. S., \& Hollweg, J. V. 1995, Sol. Phys., 157, 75

Goossens, M., Erdélyi, R., \& Ruderman, M. S. 2011, Space Sci. Rev., 158, 289

Karami, K., \& Bahari, K. 2010, Sol. Phys., 263, 87

Karami, K., \& Bahari, K. 2012, ApJ, 757, 186

Kruskal, M., \& Tuck, J. L. 1958, Proc. R. Soc. London A, 245, 222

Nakariakov, V. M., Ofman, L., Deluca, E. E., Roberts, B., \& Davila, J. M. 1999 Science, 285, 862

Parker, E. N. 1974, ApJ, 191, 245

Roberts, P. H. 1956, ApJ, 124, 430

Ruderman, M. S. 2007, Sol. Phys., 246, 119

Ruderman, M. S. 2015, A\&A, 575, A130

Ruderman, M. S., \& Erdélyi, R. 2009, Space Sci. Rev., 149, 199

Ruderman, M. S., \& Scott, A. 2011, A\&A, 529, A33

Ruderman, M. S., Verth, G., \& Erdélyi, R. 2008, ApJ, 686, 694

Ryutov, D. D., \& Ryutova, M. P. 1976, Sov. Phys. - JETP, 43, 491

Sakurai, H., Goossens, S., \& Hollweg, Y. 1991, Sol. Phys., 133, 227

Shafranov, V. D. 1957, J. Nucl. Energy II, 5, 86

Terradas, J., \& Goossens, M. 2012, A\&A, 548, A112

van Doorsselaere, T., Nakariakov, V. M., \& Verwichte, E. 2007, A\&A, 473, 959

van Doorsselaere, T., Birtill, D. C. C., \& Evans, G. R. 2009, A\&A, 508, 1485

Verth, G., \& Erdélyi, R. 2008, A\&A, 486, 1015

Verth, G., Erdélyi, R., \& Jess, D. B. 2008, ApJ, 687, L45

Verwichte, E., Nakariakov, V. M., Ofman, L., \& Deluca, E. E. 2004, Sol. Phys., 223, 77 